\title{
UPS System: How Can Future Technology and Topology Improve the Energy Efficiency in Data Centers?
}

\author{
M. Milad \\ Department of Electrical Engineering and \\ Electronics Research \\ Brunel University London, UK \\ Muftah.Milad@brunel.ac.uk
}

\author{
M. Darwish \\ Senior Lecturer \\ Department of Electrical Engineering and \\ Electronics Research \\ Brunel University London, UK \\ Mohamed.Darwish@brunel.ac.uk
}

\begin{abstract}
A Data Center can consist of a large group of networked servers and associated power distribution, networking, and cooling equipment. All these applications consume enormous amounts of energy which result in a significant increase in energy inefficiency problems. One of the causes of Data Center energy inefficiency power distribution is from the uninterruptible power supply (UPS). The UPS system is an alternate or backup source of power linking between mains power supply and end critical loads in order to provide back-up power and protection for the sensitive load. This study attempts to answer the question of how can future UPS topology and technology improve the efficiency of Data Center. In order to analyze the impact of different UPS technologies and their operating efficiencies, a model for a medium size Data Centre is developed, and load schedules and worked schematics were created to analyze and test the components of each of the UPS system topologies.
\end{abstract}

Index Terms: Data Center, Energy efficiency, Flywheel UPS system, Static UPS system, uninterruptible power supply (UPS)

\section{INTRODUCTION}

The uninterruptible power supply (UPS) system is an alternate or backup source of power, linking between mains power supply and end critical loads, to provide back-up power and to protect the sensitive load against line frequency variations [1]. The main UPS systems deployed in Data Center applications are Static, Flywheel, and DRUPS (Diesel Rotary UPS) [1]-[2]. However, the focus of this work is on the Static and Flywheel UPS systems. It analyzes the operating efficiencies of the different UPS technologies on the overall Power Usage Effectiveness (PUE) of the Data Center. The PUE is presented as a fraction whereby the total facility power inclusive of all losses is divided by the power consumed by the Data Centre IT equipment. If the PUE is equal to 1 this means a perfect efficient facility, if the given value is above 1, this means a lower facility efficiency [3]. However, the value will always be greater than 1 but the closer it is to 1 the better the Data Centre is perceived in terms of energy efficiency. Therefore, in order to analyze the different UPS technologies and their operating efficiencies a model for a medium size Data Center is developed based on a Data Center designed to provide $1500 \mathrm{~kW}$ of cooling capacity to the Data Halls and hence support $1500 \mathrm{~kW}$ of IT load. The electrical infrastructure topology to be adopted is configured to ' $2 \mathrm{~N}$ ' redundancy configuration for each of the two UPS systems technologies (Static and Flywheel), where 'N' stands for the number of UPS modules that are required to supply power to Data Center. The ' $2 \mathrm{~N}$ ' redundancy means the load of the Data Center is shared between two UPS systems on independent paths so that if one fails, the other will still supply the data center's critical load [4]. In addition, the entire critical load will be less than one half of the total UPS capacity installed. Computer based software programs such as the Romonet are used to offer a predictive modeling tool for the Data Center in order to evaluate how it would operate under different conditions. The system is designed in accordance with the Uptime Institute Tier 3 requirements, the Uptime Institute specify that all of the IT equipment is dual chorded or powered in that it can accept both an A and B supply from alternate sources [4].

\section{DESIGN DESCRIPTION}

An electrical model for a medium size Data Center is designed to provide $1500 \mathrm{~kW}$ of cooling capacity to the Data Halls. The Data Centre selected is assumed to be of the modular type operating on free air cooling which has been calculated to be available for $99 \%$ of the year in London based on Ashrae weather data. The designed IT load is $1500 \mathrm{~kW}$, which is the maximum cooling available from the mechanical systems. This, in turn, is the maximum power density of the facility. The electrical infrastructure topology adopted is configured to ' $2 \mathrm{~N}$ ' redundancy configuration for each of the two UPS systems technologies Static and Flywheel type. The system is designed in accordance with the Uptime Institute Tier 3 requirements. Table I outlines the loading accounted for when sizing the UPS. 
TABLE I

Electrical Loading Summary for Data Center

\begin{tabular}{|l|c|c|c|c|c|}
\hline Units & KW & KW & KVA & A & Remarks \\
\hline Total IT Load & 1500 & & & & \\
\hline $\begin{array}{l}\text { Data Center } \\
\text { Ventilation (Fan } \\
\text { Load) }\end{array}$ & 150 & & & & \\
\hline Total UPS Load & & 1.654 & 1.838 & 2.653 & $\begin{array}{c}\text { Assume } \\
\text { P.F }=0.9\end{array}$ \\
\hline $\begin{array}{l}\text { Total UPS Input } \\
\text { Load (incl. charging } \\
\text { current \& losses) }\end{array}$ & 1.997 & 2.264 & 3.268 & $\begin{array}{c}\text { UPS } \\
\text { charging } \\
\text { current } \\
15 \% \\
\text { UPS } \\
\text { Losses } \\
5 \%\end{array}$ \\
\hline $\begin{array}{l}\text { Total Non-UPS } \\
\text { Mechanical Cooling } \\
\text { Load }\end{array}$ & & 0 & 1 & 1 & $\begin{array}{c}\text { Assume } \\
\text { P.F }=0.9\end{array}$ \\
\hline Total Load & & 1.998 & 2.265 & 3.269 & \\
\hline
\end{tabular}

\section{A. $\quad$ Static UPS system,(Double conversion Type)}

The preferred static UPS technology used in this work is the Double Conversion Static UPS technology which is predominantly used in the Data Center Industry and uses insulated-gate bipolar transistor (IGBT) for power conversion [5]. The UPS selected for this design is a transformerless system and has the capability to operate in the Eco mode which is one of the several UPS modes of operation that can improve Data Center efficiency. In this UPS mode there are two main paths that can supply the load, the online (double conversion) path and the bypass path [6]. Hence the efficiency of the Static UPS has been derived measuring the UPS losses in double conversion mode, based on an 1.1MVA system around which the Data Centre in this study has been designed. As in Fig. 1, higher efficiency of 6-10\% could be achieved with the Static UPS by operating it in the Eco mode.

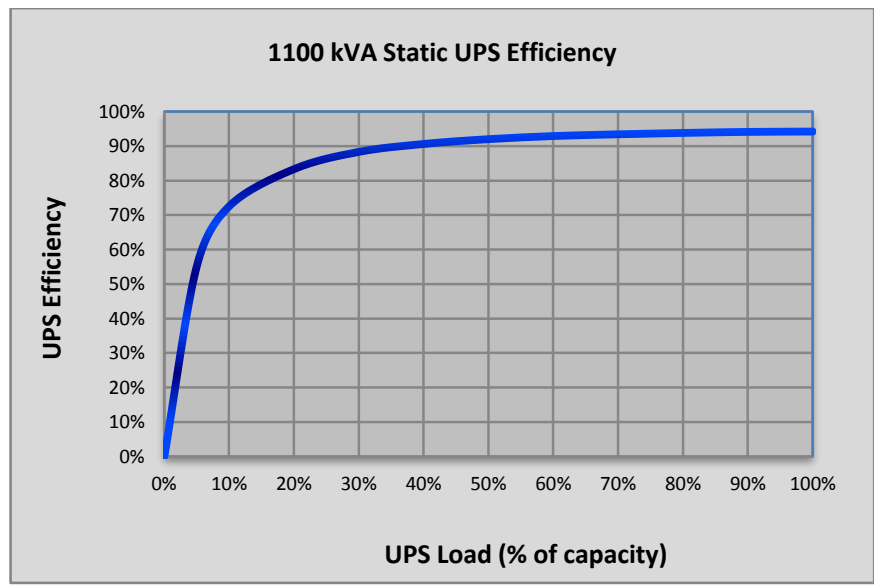

Fig. 1. Static UPS Efficiency Curve

Fig. 1 clearly indicates the efficiency of this system along the complete spectrum of loads from $5 \%$ up to maximum capacity. Considering a $2 \mathrm{~N}$ redundancy configuration at maximum capacity, this essentially is reflected as $50 \%$ loading on the UPS at maximum capacity which would result in losses of $8 \%$ on this Static UPS. However, large heat losses are experienced at low loading. To overcome this event, modular UPS systems such as the 1.1MVA system studied, can operate a UPS module management programmed where if the load is only $100 \mathrm{KVA}$, only three of the four $225 \mathrm{kVA}$ UPS modules will run in double conversion, with the other module switching to double conversion immediately, if there is a load increase or power disturbance is experienced.

The system selected is scalable in blocks of $275 \mathrm{kVA}$ up to a maximum of $1100 \mathrm{kVA}$; the UPS requirement for the design adopted requires four UPS $1100 \mathrm{kVA}$ modules. Two UPS are paralleled on "A" side and the other two UPS are paralleled on side " $\mathrm{B}$ " for the ' $2 \mathrm{~N}$ ' redundancy configuration. Table II details the output findings from the Static UPS System (double conversion type) in the ' $2 \mathrm{~N}$ ' redundancy configuration system and the calculations undertaken for the Data Center designed to provide $1500 \mathrm{~kW}$ of IT load.

TABLE II

Static UPS Findings in 2N Redundancy Configuration

\begin{tabular}{|l|c|c|c|c|}
\hline IT Load \% & $100 \%$ & $75 \%$ & $50 \%$ & $25 \%$ \\
\hline Load KW & 1500 & 1125 & 750 & 375 \\
\hline PUE & 1.27 & 1.34 & 1.48 & 1.91 \\
\hline UPS Load & $41.8 \%$ & $32.1 \%$ & $22.4 \%$ & $12.6 \%$ \\
\hline UPS Efficiency & $90.88 \%$ & $88.83 \%$ & $85.06 \%$ & $76.21 \%$ \\
\hline Impact on PUE & 0.11 & 0.14 & 0.21 & 0.41 \\
\hline
\end{tabular}

The $2 \mathrm{~N}$ redundancy configuration $100 \%$ loading will only ever represent a $50 \%$ loading on the site UPS as there will always be a minimum of 50\% redundancy [4]-[5]. The impact this redundancy has on efficiency is shown in Table II with the UPS efficiency at $25 \%$ site load running at approximately $76.2 \%$, i.e. $23.8 \%$ losses, and a maximum UPS double conversion type efficiency peaking at $90.88 \%$. Fig. 2 shows the Static UPS Efficiencies in ' $2 \mathrm{~N}$ ' redundancy configuration at part load.

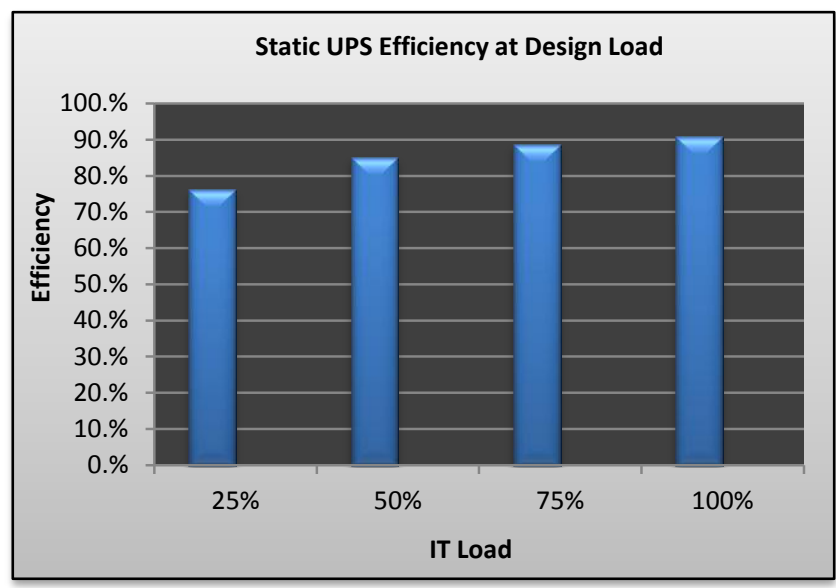

Fig. 2. Static UPS System at Part Load

\section{B. Flywheel UPS System Type}

A percentage of Data Center operators opposed to the traditional static UPS system with its requirement of large 
battery capacity have embraced flywheel technology [7]. The flywheel technology performs as a 'mechanical battery' simply converting and storing electrical energy in kinetic energy. The stored energy is used in the form of electrical power when it is required during a utility outage [7]-[8]. The system that has been selected for this comparative study is a market leader in the flywheel technology that has been successfully deployed in Data Center applications. The flywheel UPS system examined is based on a design in which a $1000 \mathrm{kVA}$ system is composed of four $250 \mathrm{kVA}$ flywheels. The power electronics losses are lower than those of the double conversion Static System. Also the additional air conditioning and battery rooms requirements for Static UPS are not needed when using flywheel UPS system. The Flywheel UPS investigated provided relatively high efficiency even at low loading. Fig. 3 clearly shows that the Flywheel UPS can achieve an efficiency of $91.2 \%$ at $20 \%$ load, while in the double conversion this level of efficiency of $92 \%$ is not gained until around $50 \%$ load.

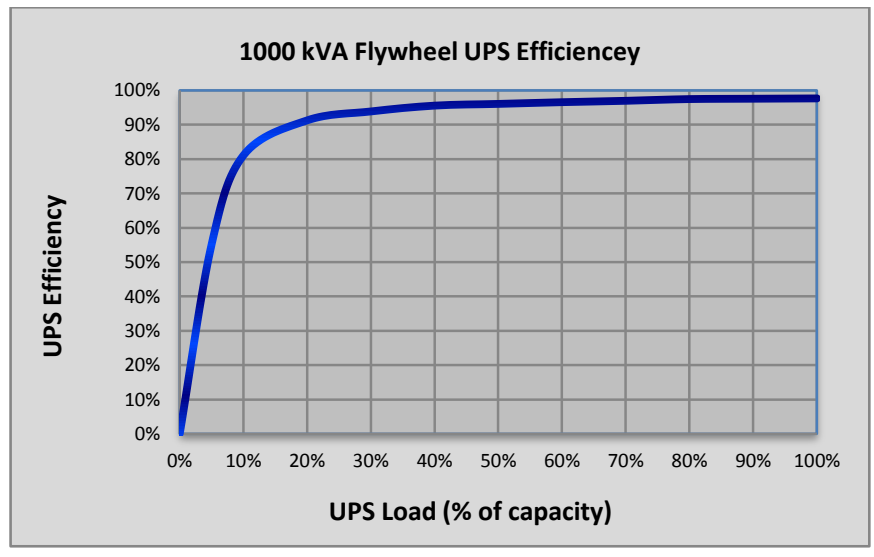

Fig. 3. Flywheel UPS Efficiency curve

In this study the UPS requirements for the ' $2 \mathrm{~N}$ ' redundancy configuration design require eight UPS 250kVA modules; four UPS are paralleled on "A" side and another four UPS paralleled on side "B". Table III details the output findings from the Flywheel UPS System in ' $2 \mathrm{~N}$ ' redundancy configuration and the calculations undertaken for the Data Center designed to provide $1500 \mathrm{~kW}$ of IT load.

TABLE III

Flywheel UPS Findings in 2N Redundancy Configuration

\begin{tabular}{|l|c|c|c|c|}
\hline IT Load \% & $100 \%$ & $75 \%$ & $50 \%$ & $25 \%$ \\
\hline Load KW & 1500 & 1125 & 750 & 375 \\
\hline PUE & 1.20 & 1.26 & 1.36 & 1.76 \\
\hline UPS Load & $45.8 \%$ & $35.2 \%$ & $24.6 \%$ & $13.8 \%$ \\
\hline UPS Efficiency & $95.79 \%$ & $94.68 \%$ & $92.40 \%$ & $81.50 \%$ \\
\hline Impact on PUE & 0.05 & 0.06 & 0.10 & 0.30 \\
\hline
\end{tabular}

Table III summarizes the results for Flywheel UPS parameters. The calculations were carried out on a ' $2 \mathrm{~N}$ ' redundancy configuration. The results on Table III have been measured across complete spectrum of loading on the site to enable comparison with the alternative technologies. Fig. 4 shows the Flywheel UPS efficiencies in ' $2 \mathrm{~N}$ ' redundancy configuration at part load.

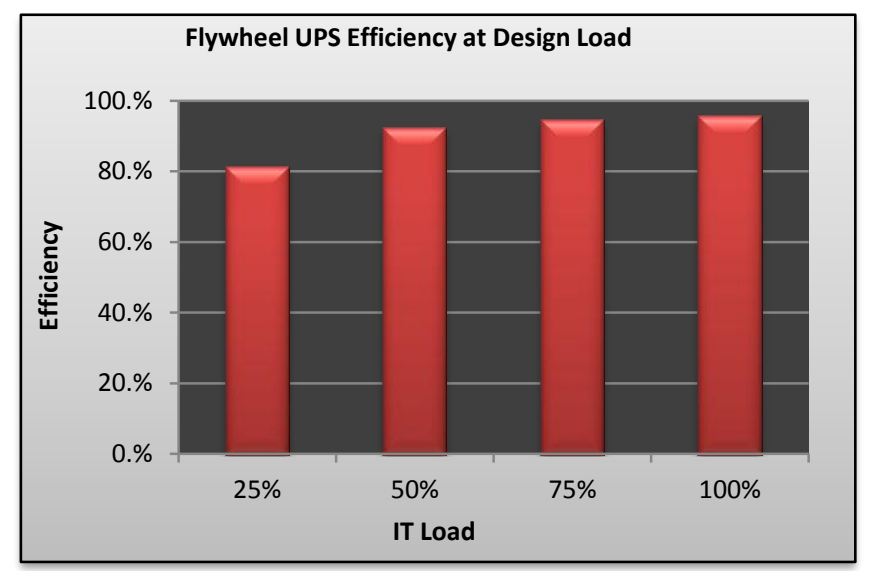

Fig. 4. Flywheel UPS System at Part Load

The efficiency profile of the Flywheel UPS is $92.40 \%$ efficiency at $50 \%$ IT load, because any system configured to operate with $100 \%$ redundancy can only ever achieve a peak loading of $50 \%$ if the load is balanced evenly across both distribution paths [4]-[7]. And that is an interesting where should result in energy savings.

\section{RESULTS AND DISCUSSION}

Comparing both the Flywheel UPSs and Double Conversion Static UPSs used in the analysis, the calculations in ' $2 \mathrm{~N}$ ' redundancy configuration UPS efficiency show that at $50 \%$ capacity the efficiency of Flywheel technology is $92.4 \%$, whereas the Double Conversion Static UPS only achieves $85.1 \%$, and the efficiency of Flywheel technology at $100 \%$ capacity is $95.7 \%$, whereas the Double Conversion Static UPS only achieves $90.8 \%$ at $100 \%$ capacity load, (see Fig. 5). Thus, the Flywheel UPS is more efficient than the Static type for the ' $2 \mathrm{~N}$ ' redundancy configuration.

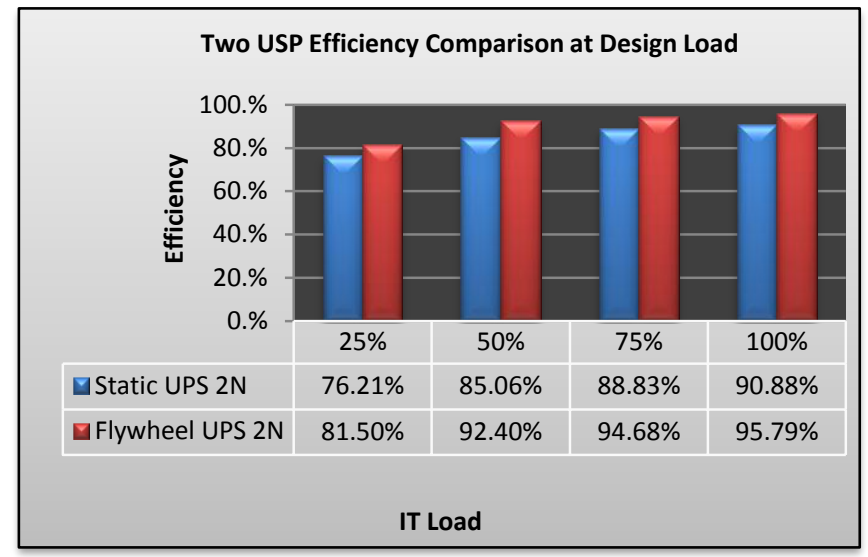

Fig. 5. 2N configuration Efficiency Comparison between two Technologies at Design Load 


\section{CONCLUSION}

This study reveals, that flywheel based UPS systems, provide dramatically better efficiency across the board than conventional double-conversion UPS with batteries, based mainly on the two-step process of converting utility power from $\mathrm{AC}$ to $\mathrm{DC}$, and then from $\mathrm{DC}$ to $\mathrm{AC}$. This higher efficiency meets the power quality target.

\section{ACKNOWLEDGEMENTS}

The author acknowledges the technical support to Jonathon Harte, at RED Engineering Design Oxford, UK, in publishing this paper.

\section{REFERENCES}

[1] S. Bekiarov, and A. Emadi, Uninterruptible power supplies: classification, operation, dynamics, and control. 17th IEEE Applied Power Electronics Conference Proceedings, Dallas, TX, March 2002, pp. 597-604.

[2] R. Hintemann, S. Pfahl. Energy Efficiency in the Data Center, a Guide to the Planning, Modernization and Operation of Data Centers, Volume 2, BITKOM, 2008.

[3] I. Bitterlin, Exploring the concept of comparative PUE, London: ARK Continuity. 2009.

[4] W. Pitt Turner, P. John, H. Seader, G. Brill, Tier Classifications Define Site Infrastructure Performance, White Paper, uptime Institute, Inc 2008.

[5] M. ton, B. Fortenbury, High Performance Buildings: Data Centers, Uninterruptible Power Supplies (UPS), December, by Ecos and EPRI, 2005.

[6] N. Rasmussen, . Eco-mode: Benefits and risks of Energy -saving mode of UPS Operation. White Paper 157, 2012.

[7] I. F. Bitterlin, Flywheel Energy Storage: an alternative to batteries in UPS System. Prism Power Ltd, UK: Watford, 2009,

[8] Active Power. Reliability Assessment of Integrated Flywheel UPS versus Double-Conversion UPS with Batteries. Austin: Active Power Limited. White Paper 103, 2011. 